\title{
RISK ASSESSMENT AND ANAESTHESIA IMPLICATIONS IN A CASE OF HYPERTROPHIC OBSTRUCTIVE CARDIOMYOPATHY FOR NON CARDIAC SURGERY
}

\author{
R. LEENA SHEKINAH ${ }^{\mathrm{a} 1}$, S.K. MASTHAN SAHEB ${ }^{\mathrm{b}}$ AND P. MRUNALINI ${ }^{\mathrm{c}}$ \\ ${ }^{a b c}$ Department of Anaesthesiology, NRI Academy of Medical Sciences, Guntur, Andhra Pradesh, India
}

\begin{abstract}
Hypertrophic Cardiomyopathy with or without left ventricular outflow tract obstruction is characterised by Asymmetric hypertrophy of the interventricular septum causing intermittent obstruction of the left venticular out flow tract. Because Hypertrophic cardiomyopathy is the most common genetic cardiovascular disease it may present to the anaesthesiologist more often than anticipated. Surgery and Anesthesia often complicate the perioperative outcome of these patients. Aim of this case report is to highlight the importance of perioperative risk assessment of the patient and proper understanding of the pathophysiology of the disease for optimal anaesthetic management and postoperative outcome. A $62 \mathrm{yr}$ old male who is a known case of Hypertrophic Obstructive Cardiomyopathy incidently diagnosed 9 yrs back had undergone ORIF with plating of left fibula following a trauma 5 yrs back under spinal anaesthesia. He has now been posted for cannulated cancellous screw removal.
\end{abstract}

KEYWORDS: Hypertrophic Obstructive Cardiomyopathy

Hypertrophic Cardiomyopathy is the most common of the genetic cardiovascular diseases inherited in an autosomal dominant manner (Sahoo et al., 2010). The inciting factor is myocardial hypertrophy which involves septum and LV free wall (Bhalerao et al., 2017). This asymmetric septal hypertrophy leads to a variable pressure gradient between the apical LV chamber and the LV outflow tract (LVOT). The LVOT obstruction leads to increased LV pressure which creates a vicious cycle of hypertrophy, begetting increased LVOT obstruction.

\section{CASE REPORT}

A 62 year old male who is a known case of HOCM incidently diagnosed 9 year back presented to the Orthopedic outpatient department for Cannulated Cancellous screw removal. He underwent ORIF and plating of the left fibula following a trauma 5 yrs back under Subarachnoid block. He also gives a history of progressively worsening dyspnoea grade I-III over the last 7 months with a couple of episodes of syncope. He is a newly diagnosed type 2 Diabetic and a known case of Hypothyroidism on regular treatment since $10 \mathrm{yrs}$. He is an ex-smoker and not an alcoholic. His father and father's brother had both passed away from cardiac disease in their 50's. On physical examination pulse rate was 84 beats per minute, sinus rhythm, normal volume in the right radial artery, blood pressure of $150 / 100 \mathrm{mmHg}$ in the right brachial artery in supine position. The systemic examination of the Cardiovascular system revealed a high pitched, crescendo-decresendo mid systolic murmur heard at the left lower sterna border, with no radiation to the carotids. The rest of the systemic examination was nill significant. Chest xray showed cardiomegaly with a cardiothoracic ration of 0.6. 12 lead Electrocardiogram showed sinus rhythm with evidence of left atrial and ventricular hypertrophy with strain pattern. Echocardiography revealed asymmetric septal hypertrophy, SAM + , severe LVOT obstruction with gradient of $143 \mathrm{mmHg}$ with mild $\mathrm{MR}$, mild TR, mild PAH with estimated RVSP of +33RAP $\mathrm{mmHg}$ and a IV septal/LVWD ratio of $1.9 \mathrm{cms}$. The ejection fraction was $67 \%$ with no RWMA or LA/LV clots. His LVOT gradient had increased from 50mmhg in 2008 to $90 \mathrm{mmhg}$ in 2013.

The pt was accepted for anaesthesia under grade IV ASA. He was posted for left ankle cancellous screw removal for which Local anaesthesia was adviced.

\section{DISCUSSION}

Hypertrophic cardiomyopathy is characterized by asymmetric hypertrophy of the intervetricular septum causing dynamic obstruction of the left ventricular outflow tract. The pathophysiologic determinants of clinical course and disease progression in HCM include (Livin et al., 2006):

1. Dynamic obstruction to LV outflow due to mitral valve systolic anterior motion and hypertrophied ventricular septal contact

2. Diastolic dysfunction, associated with increased filling pressures resulting from impaired LV relaxation and filling of a hypertrophied and noncompliant LV. 
3. Impaired coronary vasodilator reserve and myocardial ischemia

4. Supraventricular and ventricular tachyarrhythmias (e.g., atrial fibrillation $[\mathrm{AF}]$ and ventricular tachycardia/ fibrillation)

In a normal healthy individual as such no LVOT gradient is present.

A gradient of $0-30 \mathrm{mmHg}$ is considered mild, $30-50 \mathrm{mmHg}$ is moderate, $>50 \mathrm{mmHg}$ is high risk.

Decrease in venous return and systemic vascular resistance or increase in the myocardial contractility can worsen the LVOT Obstruction. These pt are highly prone 1. to arrhythmias like atrial fibrillation and ventricular tachycardia which can at times prove to be fatal.

Hypertrophy of the IV septum is considered when the IV septal wall/ LVPW thickness is $>1.1 \mathrm{~cm}$ (Schvartzman et al., 2000).

IV septal wall/LVPW thickness ratio:

- Normal :- 0.6-1.0

- $\quad$ Mild Hypertrophy:-1.1- 1.3

- Moderate Hypertrophy:-1.4-1.6

- $\quad$ Severe Hypertrophy:- $>1.7$

Although considerable data are available regarding cardiovascular risks for patients with atherosclerotic coronary artery disease undergoing anesthesia for noncardiac surgery, there is little information available regarding such risks for $\mathrm{HCM}$ patients. This is probably largely because of the relative infrequency with which patients with a confirmed HCM diagnosis are encountered by anesthesiologists. Nevertheless, HCM patients (with or without LV outflow obstruction) undergoing noncardiac surgery not uncommonly experience adverse perioperative outcomes. Reported events include congestive heart failure, myocardial ischemia, systemic hypotension, and supraventricular or ventricular arrhythmias. Length and type of surgery (major vs. minor) are predictors of adverse cardiac outcome in HCM patients.

The risk assessment for HCM takes into consideration six major risk factors (Braunwald's Heart Disease):-

1. Previous cardiac arrest/ sustained ventricular tachycardia.

2. Non sustained ventricular tachycardia.

3. Extreme left ventricular hypertrophy with high LVOT gradient.

4. Unexplained syncope
5. Abnormal blood pressure response

6. Family history of sudden cardiac death.

Based on the patients history of worsening dyspnoea, H/o syncope family history of sudden cardiac deaths and clinical examination the pt was classified as high risk and the previously administered route of subarachnoid block was avoided.

Anaesthetic technique and the perioperative management of these patients must aim to maintain haemodynamic stability, adequate preload and afterload. Events that increase the LVOT gradient like:

Increased myocardial contractility due to $\beta$ adrenergic stimulation

2. Decrease in preload due to hypovolemia, vasodilators, IPPV, tachycardia.

3. Decrease in afterload due to hypovolemia.

must be avoided.

Administration of general anaesthesia causes Hypotension due to decreased systemic vascular resistance and intubation response increases sympathetic stimulation and tachycardia. Profound hypotension occurs following peripheral sympathetic blockade after neuraxial anaesthesia. As a treatment of hypotension, use of $\beta$ adrenergic agonist - ephedrine, dopamine, dobutamine increases myocardial contractility \& tachycardia causing LVOT obstruction. Therefore General and Spinal/Epidural anaesthesia are relatively contraindicated in this patient.

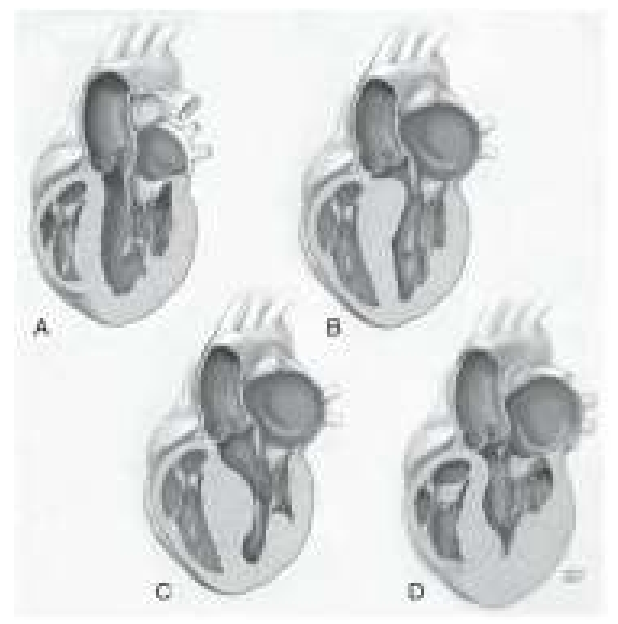

Figure 1: Normal Heart and Phenotypic variants of Hypertrophic Cardiomyopathy. [A] Normal Heart.

[B] Isolated basal septal hypertrophy. [C] Midventricular septal hypertrophy. [D] Apical hypertrophy 


\section{CONCLUSION}

Even though the patient is a known case of HOCM and had received spinal anaesthesia 5 yrs back, the same route of treatment was not recommended in view of:

1. Clinically worsening dyspnoea.

2. High ratio of IV Septal wall thickness to LV wall

3. Increased LVOT gradient.

Hence the safest route was taken and the procedure was done under local anaesthesia.

\section{REFERENCES}

Sahoo R.K., Dash S.K., Raut P.S., Badole U.R. and Upasani C.B., 2010. Perioperative anesthetic management of patients with hypertrophic cardiomyopathy for noncardiac surgery: A case series. Ann Card Anaesth, 13: 253-6.
Bhalerao P.M., Adsule P.B. and Marathe R.M., 2017. Anaesthetic management of a patient with hypertrophic obstructive cardiomyopathy posted for emergency lower segment caesarean section. J. Obstet Anaesth Crit Care, 7: 103-5.

Livin C.P., Michael E.B. and Barry J.M., 2006. Hypertrophic Anaesthesiology, 104(1): 183-192.

Schvartzman P.R., Fuchs F.D., Mello A.G., Coli M., Schvartzman M. and Moreira L.B., 2000. Normal values of echocardiographic measurements. A population-based study. Arq. Bras. Cardiol, 75(2): 111-114.

Braunwald's Heart Disease. A Textbook of Cardiovascular Medicine. Tenth Edition page 1582. 